\title{
Finite Temperature Phase Diagram of a Two-Component Fermi Gas with Density Imbalance
}

\author{
Lianyi He*, Meng Jin ${ }^{\dagger}$ and Pengfei Zhuang $¥$ \\ Physics Department, Tsinghua University, Beijing 100084, China
}

\begin{abstract}
We investigated possible superfluid phases at finite temperature in a two-component Fermi gas with density imbalance. In the frame of a general four-fermion interaction theory, we solved in the BCS region the gap equations for the pairing gap and pairing momentum under the restriction of fixed number densities, and analyzed the stability of different phases by calculating the superfluid density and number susceptibilities. The homogeneous superfluid is stable only at high temperature and low number asymmetry, the inhomogeneous LOFF survives at low temperature and high number asymmetry, and in between them there exists another possible inhomogeneous phase, that of phase separation. The critical temperatures and the orders of the phase transitions among the superfluid phases and normal phase are calculated analytically and numerically. The phase diagram we obtained in the temperature and number asymmetry plane is quite different from the one in temperature and chemical potential difference plane for a system with fixed chemical potentials.
\end{abstract}

PACS numbers: 03.75.Ss, 05.30.Fk, 74.20.Fg, 34.90.+q

\section{INTRODUCTION}

Recently, the superfluidity in a two-component Fermi gas with population imbalance promoted great interest in both experimental and theoretical studies. The key point is the ground state of fermion pairing between different species with mismatched Fermi surfaces, and it is directly related to the studies of atomic Fermi gas with population imbalance 1, 2, 3, 3, 45, 6, 7, 8, 9, 10, 11, 12, 13, 14, 15, 16, 17, 18], superconductivity with Zeeman splitting 19, 20, 21, 22], isospin asymmetric nuclear matter [23, 24, 25) and dense QCD matter [26, 27, 28, 29, 30]. In the investigation, two exotic states, the homogeneous and isotropic Sarma [19] or breached pairing [31, 32] state and the inhomogeneous and anisotropic Larkin-OvchinnikovFudde-Ferrell (LOFF) 20, 21, 22, 33] state, are especially concerned. While these two interesting states have never been observed experimentally, the recent progress in the study of atomic Fermi gas 34, 35, 36] may provide us a way to realize and observe them.

There are two crucial problems related to the Sarma state due to the existence of gapless fermionic excitation. One problem is its thermodynamical instability compared with the fully gapped BCS state, when the effective chemical potentials for the two species are fixed. This is called Sarma instability [19]. It is now accepted that the Sarma instability can be avoided in systems under some physical constraints such as fixed particle numbers [23, 37] instead of fixed chemical potentials, long range gauge interaction with charge neutrality requirement 27], a proper finite range attractive interaction [32], and in the strong coupling BEC region [3, 38]. The second problem for the Sarma state is

\footnotetext{
${ }^{*}$ Email address: hely04@mails.tsinghua.edu.cn

${ }^{\dagger}$ Email address: jin-m@mail.tsinghua.edu.cn

${ }^{\ddagger}$ Email address: zhuangpf@mail.tsinghua.edu.cn
}

its negative superfluid density [3, 39] and negative number susceptibility [3]. The former leads to negative Meissner mass squared [40, 41] for charged systems and indicates that the LOFF state is probably energetically favored than the Sarma state 42, 43], and the latter shows that the superfluid-normal phase separation[1] is also more stable than the Sarma state.

The above discussed negative superfluid density and number susceptibility are obtained in weak coupling BCS region and at zero temperature. Recently, it is argued that the Sarma phase will be free from negative superfluid density and number susceptibility in strong coupling BEC region [3, 5, 38], and both experimental and theoretical studies support that the normal-superfluid phase separation is energetically favored around the unitary region [5, 36].

In this paper we are interested in the BCS region where the LOFF phase is shown to be energetically favored[5, 10]. An important and interesting phenomenon in this region is the enhanced pairing correlation at finite temperature [44, 45]. The pairing gap is not a monotonous function of temperature and its maximum is located not at zero but at finite temperature. Especially, for a large number asymmetry, the superfluidity appears only at finite temperature. This strange and interesting phenomenon was found in the studies of isospin asymmetric nuclear matter 23, 24], two flavor color-superconducting quark matter [27], and general breached pairing superfluidity [37]. However, it is recently found that the superfluid density of the homogeneous Sarma phase is negative at low temperature and becomes positive only in a temperature window near the critical temperature $T_{c}[44,446]$. As a result, inhomogeneous phases such as LOFF phase can enter the low temperature region, and the phase structure should be re investigated.

The purpose of this paper is to analyze the phase structure of a two-component Fermi gas with density imbalance in the BCS regime. Our analysis is based on the 
mean field approximation which is believed to be a good treatment in the BCS regime even at finite temperature. We take LOFF phase into account. For the sake of simplicity, we consider the simplest pattern of LOFF phase, namely, the single plane wave LOFF state or the so-called FF state. While the consideration of a general LOFF state is complicated, we will argue that the phase structure with the FF state will not be changed qualitatively. Due to the thermal excitation, we will not distinguish between the gapless and gapped phases and simply call them homogeneous superfluid (HS).

After a brief review of the theoretical frame for the attractive two-component Fermi gas with population imbalance in Section [I] we calculate in Section [II] the superfluid density and number susceptibility as a function of temperature at fixed population imbalance and obtain the turning temperature where the superfluid density and number susceptibility both change sign. In Section IV we include the LOFF state and separate the homogeneous region from the possible LOFF region. By calculating in Section $\nabla$ the number susceptibility $\chi$ for the LOFF state, we further distinguish the stable LOFF region at high population imbalance from unstable LOFF region at low population imbalance. We obtain the phase diagram in the temperature and population imbalance plane in Section [V] We summarize in Section VI] The natural unit of $c=\hbar=k_{B}=1$ is adopted through the paper.

\section{FORMALISM}

The physical system we are interested in in this paper is an infinite system composed of two species of fermions with attractive interaction in three dimensional free space. Generally, the system can be modelled by the Lagrangian density

$$
\mathcal{L}=\sum_{\sigma=\uparrow, \downarrow} \psi_{\sigma}^{\dagger}\left(i \partial_{t}+\frac{\nabla^{2}}{2 m}+\mu_{\sigma}\right) \psi_{\sigma}+g \psi_{\uparrow}^{\dagger} \psi_{\downarrow}^{\dagger} \psi_{\downarrow} \psi_{\uparrow},
$$

where $\psi_{\sigma}(x)$ are fermion fields for the two species denoted by $\uparrow$ and $\downarrow$ with space-time $x=(t, \mathbf{x}), g$ is the coupling constant, $m$ is the fermion mass, and $\mu_{\uparrow}$ and $\mu_{\downarrow}$ are the chemical potentials.

For attractive coupling $g$ we can perform an exact Stratonovich-Hubbard transformation to introduce the pair field $\Phi \sim g \psi_{\downarrow} \psi_{\uparrow}$ and its complex conjugate $\Phi^{*} \sim$ $g \psi_{\uparrow}^{\dagger} \psi_{\downarrow}^{\dagger}$. With the Nambu-Gorkov field defined as $\Psi=$ $\left(\psi_{\uparrow}, \psi_{\downarrow}^{\dagger}\right)^{T}$, the partition function can be expressed as

$$
Z=\int\left[d \Psi^{\dagger}\right][d \Psi]\left[d \Phi^{*}\right][d \Phi] e^{\int_{0}^{\beta} d \tau \int d^{3} \mathbf{x}\left(\Psi^{\dagger} \mathcal{K} \Psi-\Phi^{*} \Phi / g\right)}
$$

in the imaginary time $(\tau=i t)$ formalism of finite temperature field theory, where $\beta$ is the inverse temperature, $\beta=1 / T$, and the kernel $\mathcal{K}$ is defined as

$$
\mathcal{K}\left[\Phi^{*}, \Phi\right]=\left(\begin{array}{cc}
-\partial_{\tau}+\frac{\nabla^{2}}{2 m}+\mu_{\uparrow} & \Phi(\tau, \mathbf{x}) \\
\Phi^{*}(\tau, \mathbf{x}) & -\partial_{\tau}-\frac{\nabla^{2}}{2 m}-\mu_{\downarrow}
\end{array}\right) .
$$

Integrating out the fermionic degrees of freedom, we obtain

$$
Z=\int\left[d \Phi^{*}\right][d \Phi] e^{-S_{e f f}\left[\Phi^{*}, \Phi\right]}
$$

with the effective action

$$
S_{\text {eff }}\left[\Phi^{*}, \Phi\right]=\int_{0}^{\beta} d \tau \int d^{3} \mathbf{x} \frac{\Phi^{*} \Phi}{g}-\operatorname{Tr} \ln \mathcal{K}\left[\Phi^{*}, \Phi\right] .
$$

For a dilute gas, we can replace the bare coupling constant $g$ by the low energy limit of the two-body Tmatrix [3],

$$
\frac{m}{4 \pi a_{s}}=-\frac{1}{g}+\int \frac{d^{3} \mathbf{p}}{(2 \pi)^{3}} \frac{1}{2 \epsilon_{p}}
$$

with the $s$-wave scattering length $a_{s}$ and fermion energy $\epsilon_{p}=\mathbf{p}^{2} /(2 m)$.

For an arbitrary scattering length $a_{s}$ at finite temperature $T$, we should take into account the contribution from the pair fluctuations and pseudogap 44] to the thermodynamics of the system. Since in this paper we focus on the BCS region where the scattering length is negative and small and the coupling is relatively weak, the effect of pair fluctuations and pseudogap can be approximately neglected, and the mean field approximation is a good approach to investigate the phase structure. In the mean field approximation, we replace the pair field $\Phi$ and its complex conjugate by their expectation values. To have a unified treatment for both the homogeneous and LOFF superfluid, the order parameter for the superfluid can be defined as

$$
\langle\Phi(x)\rangle=\Delta e^{2 i \mathbf{q} \cdot \mathbf{x}}, \quad\left\langle\Phi^{*}(x)\right\rangle=\Delta e^{-2 i \mathbf{q} \cdot \mathbf{x}},
$$

where $\Delta$ is the amplitude of the order parameter and can be taken to be real, and $2 \mathbf{q}$ is the pair momentum in single plane wave LOFF state. Obviously, $\mathbf{q}=0$ and $\mathbf{q} \neq 0$ correspond, respectively, to the HS and LOFF states.

Since a general LOFF state can be considered as a superposition of single plane wave LOFF states, it corresponds to a deeper minimum of the free energy of the system, compared with the single plane wave LOFF state. Therefore, a general LOFF state should be more stable than the simplest LOFF state, and the simplest LOFF phase, if it exists, will be replaced by a general LOFF phase. In this sense, while the details of our phase diagrams obtained in the following will be changed, the qualitative phase structure of the system will remain, when a general LOFF state is included.

After a phase transformation for the fermion fields, $\chi_{\sigma}=e^{i \mathbf{q} \cdot \mathbf{x}} \psi_{\sigma}$, the thermodynamic potential in mean field approximation can be evaluated as a summation of quasiparticles 43 ]

$$
\begin{aligned}
\Omega= & -\frac{m \Delta^{2}}{4 \pi a_{s}}-\Delta^{2} \int \frac{d^{3} \mathbf{p}}{(2 \pi)^{3}}\left(\frac{1}{E_{p}+\xi_{p}}-\frac{1}{2 \epsilon_{p}}\right) \\
& -\frac{1}{\beta} \int \frac{d^{3} \mathbf{p}}{(2 \pi)^{3}}\left[\ln \left(1+e^{-\beta E_{A}}\right)+\ln \left(1+e^{-\beta E_{B}}\right)\right],
\end{aligned}
$$


where $E_{A}$ and $E_{B}$ are the quasi-particle energies

$$
\begin{aligned}
& E_{A}=E_{p}+\delta \mu+\mathbf{p} \cdot \mathbf{q} / m, \\
& E_{B}=E_{p}-\delta \mu-\mathbf{p} \cdot \mathbf{q} / m
\end{aligned}
$$

with $\xi_{p}=\left(\mathbf{p}^{2}+\mathbf{q}^{2}\right) /(2 m)-\mu, E_{p}=\sqrt{\xi_{p}^{2}+\Delta^{2}}$, and average chemical potential $\mu=\left(\mu_{\uparrow}+\mu_{\downarrow}\right) / 2$ and chemical potential difference $\delta \mu=\left(\mu_{\downarrow}-\mu_{\uparrow}\right) / 2$. Note that, since the replacement of the bare coupling $g$ by the s-wave scattering length $a_{s}$, the ultraviolet divergence in the first momentum integration in (8) is removed, and there is no need to introduce a momentum cutoff.

The thermodynamic potential obtained is a function of $T, \mu_{\uparrow}$ and $\mu_{\downarrow}$ with $\Delta$ and $\mathbf{q}$ initially undetermined order parameter and pair momentum of the superfluid. In the spirit of thermodynamics, the physical system is described only by $T, \mu_{\uparrow}$ and $\mu_{\downarrow}$, and $\Delta$ and $\mathbf{q}$ as functions of $T, \mu_{\uparrow}, \mu_{\downarrow}$ are determined by the minimum thermodynamic potential. The calculation of the first order derivatives of $\Omega$ with respect to $\Delta$ and $\mathbf{q}$ gives the coupled gap equations,

$$
\begin{aligned}
-\frac{m \Delta}{4 \pi a_{s}}= & \Delta \int \frac{d^{3} \mathbf{p}}{(2 \pi)^{3}}\left[\frac{1-f\left(E_{A}\right)-f\left(E_{B}\right)}{2 E_{p}}-\frac{1}{2 \epsilon_{p}}\right] \\
0= & \int \frac{d^{3} \mathbf{p}}{(2 \pi)^{3}}\left[\frac{\mathbf{p} \cdot \mathbf{q}}{q}\left(f\left(E_{B}\right)-f\left(E_{A}\right)\right)\right. \\
& \left.-q\left(1-\frac{\xi_{p}}{E_{p}}\left(1-f\left(E_{A}\right)-f\left(E_{B}\right)\right)\right)\right],(10)
\end{aligned}
$$

where we have chosen a suitable frame with the $z$ axis along the direction of the pair momentum, $\mathbf{q}=(0,0, q)$, and $f(x)$ is the Fermi-Dirac distribution function. It is easy to see that $q=0$ is a trivial solution of the gap equations, which corresponds to the homogeneous and isotropic phase. For a system with fixed numbers of species, the chemical potentials $\mu_{\uparrow}$ and $\mu_{\downarrow}$ in (10) are solved from the fermion number densities $n_{\uparrow}$ and $n_{\downarrow}$ derived by the thermodynamic relations $n_{\sigma}=-\partial \Omega / \partial \mu_{\sigma}$,

$$
n_{\uparrow}=\int \frac{d^{3} \mathbf{p}}{(2 \pi)^{3}} n_{\uparrow}(p), \quad n_{\downarrow}=\int \frac{d^{3} \mathbf{p}}{(2 \pi)^{3}} n_{\downarrow}(p),
$$

where $n_{\uparrow}(p)$ and $n_{\downarrow}(p)$ are the occupation numbers for the two species,

$$
\begin{aligned}
& n_{\uparrow}(p)=u_{p}^{2} f\left(E_{A}\right)+v_{p}^{2} f\left(-E_{B}\right), \\
& n_{\downarrow}(p)=u_{p}^{2} f\left(E_{B}\right)+v_{p}^{2} f\left(-E_{A}\right)
\end{aligned}
$$

with the coherent coefficients $u_{p}^{2}=\left(1+\xi_{p} / E_{p}\right) / 2$ and $v_{p}^{2}=\left(1-\xi_{p} / E_{p}\right) / 2$.

The gap equations (10) together with the fermion numbers (111) determine the physical values of $\Delta$ and $q$ as functions of $n_{\uparrow}$ and $n_{\downarrow}$. Generally, there exist a homogeneous solution with $q=0$ and a LOFF solution with $q \neq 0$. To determine which one is favored at fixed fermion numbers, we should compare their free energies defined as

$$
\mathcal{F}\left(n_{\sigma}, T\right)=\Omega+\mu_{\uparrow} n_{\uparrow}+\mu_{\downarrow} n_{\downarrow},
$$

and the lower one corresponds to the ground state of the system.

To explicitly describe the asymmetry between the two species, we use the total density $n=n_{\uparrow}+n_{\downarrow}$ and the number asymmetry parameter $\alpha=\left(n_{\downarrow}-n_{\uparrow}\right) /\left(n_{\downarrow}+n_{\uparrow}\right)$ as variables instead of $n_{\uparrow}$ and $n_{\downarrow}$. Without loss of generality, we suppose $\alpha \geq 0$ in the following. Like a free Fermi gas, we introduce a Fermi momentum $p_{F}$ or a Fermi energy $E_{F}=p_{F}^{2} /(2 m)$ through $n=p_{F}^{3} /\left(3 \pi^{2}\right)$. If we scale all the variables with energy dimension by $E_{F}$ and the variables with momentum dimension by $p_{F}$, the solution of the gap equations depends only on three dimensionless variables, the coupling $p_{F} a_{s}$, population imbalance $\alpha$ and the scaled temperature $T / E_{F}$. For the following numerical calculation in the BCS region with $-1<p_{F} a_{s}<0$ or $-\infty<1 /\left(p_{F} a_{s}\right)<-1$, we take $p_{F} a_{s}=-0.6$. This is a typical value for systems with BCS pairing. For example, for the pairing between ${ }^{6} \mathrm{Li}$ atoms in states $\left|F=3 / 2, m_{F}=3 / 2\right\rangle$ and $\left|F=3 / 2, m_{F}=1 / 2\right\rangle$ with scattering length $a_{s}=-2160 a_{B}$ where $a_{B}$ is the Borh radius and typical density $n=3.8 \times 10^{12} \mathrm{~cm}^{-3}$, we have $p_{F} a_{s}=-0.56$.

\section{TEMPERATURE BEHAVIOR OF THE HOMOGENEOUS PHASE}

In this section we focus on the homogeneous phase. We analyze the temperature behavior of the pairing gap, the superfluid density and the number susceptibility, and show that there should be a phase transition from homogeneous phase at higher temperature to some inhomogeneous phase at low temperature.

\section{A. Order Parameter $\Delta$}

At zero temperature, the gap and the chemical potential difference for an asymmetric system with $\alpha \neq 0$ satisfy the relation $\delta \mu>\Delta$, which can be seen directly from the number equations. This means that the homogeneous phase at $T=0$ must be a gapless superfluid [41] with the gapless branch $E_{B}$. In the BCS region, we have a positive chemical potential $\mu$ which is not far from the Fermi energy $E_{F}$, and the chemical potential mismatch $\delta \mu$ is much less than $\mu$, the two gapless nodes occur at momenta $p_{1}=\sqrt{2 m\left(\mu-\sqrt{\delta \mu^{2}-\Delta^{2}}\right)}$ and $p_{2}=\sqrt{2 m\left(\mu+\sqrt{\delta \mu^{2}-\Delta^{2}}\right)}$. The behavior of $\Delta$ is quite different from the one for symmetric BCS gap $\Delta_{0}$ with the same coupling $p_{F} a_{s}$. In weak coupling, there is an analytical relation between them [19],

$$
\Delta(\delta \mu)=\sqrt{\Delta_{0}\left(2 \delta \mu-\Delta_{0}\right)}, \quad \Delta_{0} / 2<\delta \mu<\Delta_{0}
$$


in terms of the chemical potential mismatch or [4]

$$
\Delta(\alpha)=\Delta_{0} \sqrt{1-\alpha / \alpha_{0}} \Theta\left(\alpha_{0}-\alpha\right)
$$

in terms of the population imbalance, where $\Theta(x)$ is a step function and the critical imbalance $\alpha_{0}$ is given by $\alpha_{0}=3 \Delta_{0} /\left(4 E_{F}\right)$. The solution $\delta \mu=\Delta_{0}$ corresponds to $\alpha=0$ and $\delta \mu=\Delta_{0} / 2$ to $\alpha=\alpha_{0}$.

For the symmetric system, it is well-known that the pairing gap $\Delta_{0}$ will be suppressed by thermal motion at finite temperature, as shown by the solid line marked with $\alpha=0$ in Fig.1. However, this monotonous temperature behavior is not always true for the asymmetric system. The temperature effect not only deforms and reduces the mismatched Fermi surfaces which leads to the usual suppression of the gap, but also makes the overlap region of the two species wider which favors the condensate. The competition of the two opposite effects results in a non-monotonous temperature behavior of the pairing gap. In Fig 1, we show the gap $\Delta$ as a function of temperature for different values of population imbalance $\alpha$ and at the typical BCS coupling $p_{F} a_{s}=-0.6$. In the case with weak imbalance, while the superfluidity starts still at $T=0$, it firstly goes up with increasing temperature, then drops down, and finally reaches zero at the critical temperature $T_{c}$. In the case with strong imbalance, the superfluidity starts even at a finite temperature $T_{o}>0$ and vanishes at $T_{c}$. The phase transitions at the two critical temperatures $T_{o}$ and $T_{c}$ from normal phase to HS is of second order, and the critical behavior of the gap can be conventionally expressed as [48]

$$
\begin{array}{ll}
\Delta(T)=\phi T_{o}\left(T / T_{o}-1\right)^{1 / 2}, & T \rightarrow T_{o}^{+}, \\
\Delta(T)=\varphi T_{c}\left(1-T / T_{c}\right)^{1 / 2}, & T \rightarrow T_{c}^{-},
\end{array}
$$

where $T_{o}, T_{c}$ and the dimensionless coefficients $\phi$ and $\varphi$ all depend on the population imbalance $\alpha$. When $\alpha$ exceeds the maximum value $\alpha_{H S}$ for the HS phase, there is no more homogeneous superfluidity at any temperature. In Fig 1 we have $\alpha_{H S}=0.059$.

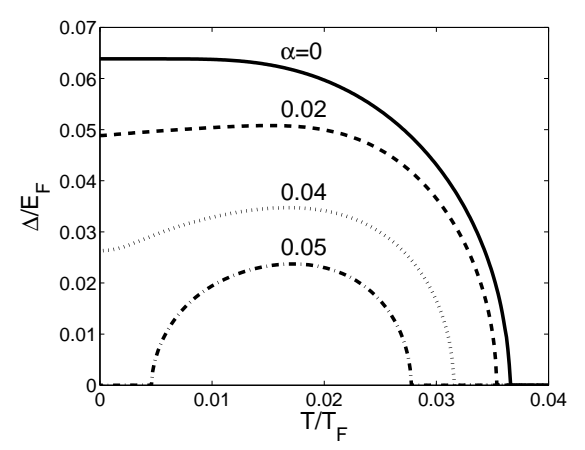

FIG. 1: The pairing gap $\Delta$, scaled by the Fermi energy $E_{F}$, as a function of $T$, scaled by the Fermi temperature $T_{F}=$ $E_{F} / k_{B}$, for different values of number asymmetry $\alpha$.

\section{B. Superfluid Density $\rho_{s}$}

When the superfluid moves with a uniform velocity $\mathbf{v}_{s}$, the condensate transforms like $\langle\Phi\rangle \rightarrow$ $\langle\Phi\rangle e^{2 i m \mathbf{v}_{s} \cdot \mathbf{x}},\left\langle\Phi^{*}\right\rangle \rightarrow\left\langle\Phi^{*}\right\rangle e^{-2 i m \mathbf{v}_{s} \cdot \mathbf{x}}$, and the supercurrent $\mathbf{j}_{s}$ and the superfluid density $\rho_{s}$ are defined via

$$
\Omega\left(\mathbf{v}_{s}\right)=\Omega(0)+\mathbf{j}_{s} \cdot \mathbf{v}_{s}+\frac{1}{2} \rho_{s} \mathbf{v}_{s}^{2}+\cdots
$$

From the introduction (17) for the pair momentum $\mathbf{q}$ and its gap equation, the supercurrent keeps zero,

$$
\mathbf{j}_{s}=\left.\frac{\partial \Omega}{\partial \mathbf{v}_{s}}\right|_{\mathbf{v}_{s}=0}=\left.m \frac{\partial \Omega}{\partial \mathbf{q}}\right|_{\mathbf{q}=0}=0
$$

and the superfluid density $\rho_{s}$ is directly related to the pair momentum susceptibility

$$
\rho_{s}=\left.\frac{\partial^{2} \Omega}{\partial \mathbf{v}_{s}^{2}}\right|_{\mathbf{v}_{s}=0}=\left.m^{2} \frac{\partial^{2} \Omega}{\partial \mathbf{q}^{2}}\right|_{\mathbf{q}=0} .
$$

From the stability condition $\partial^{2} \Omega / \partial \mathbf{q}^{2} \geq 0$ for the pair momentum q, the superfluid density controls the stability of the homogenous phase. When $\rho_{s}$ is negative, the HS state is unstable. It is necessary to note that, up to the square term, the expansions of the free energy $\mathcal{F}$ in $\mathbf{v}_{s}$ and $\mathbf{q}$ are the same as the expansions of $\Omega$. Therefore, we can safely use the definition (19) to analyze the superfluid stability for the system with fixed number densities [49].

Using the result in [39, 41], the superfluid density at finite temperature can be explicitly expressed as

$$
\rho_{s}=m n+\int_{0}^{\infty} d p \frac{p^{4}}{6 \pi^{2}}\left[f^{\prime}\left(E_{A}\right)+f^{\prime}\left(E_{B}\right)\right]
$$

with the definition $f^{\prime}(x)=d f(x) / d x$. Note that the integration term is always negative, and the asymmetry between the two species may induce a negative superfluid density $\rho_{s}$. At zero temperature and in weak coupling limit, $\rho_{s}$ can be approximately expressed as [41],

$$
\rho_{s} \approx m n\left[1-\frac{\delta \mu \Theta(\delta \mu-\Delta)}{\sqrt{\delta \mu^{2}-\Delta^{2}}}\right]
$$

It is easy to see that in the Sarma phase with $\Delta<\delta \mu, \rho_{s}$ becomes negative. This is the so called magnetic instability, since it is directly related to the negative Meissner mass squared $m_{A}^{2}=e^{2} \rho_{s} / m^{2}$ [41] if the fermions are charged. This dynamical instability implies that the LOFF state with finite pair momentum has lower free energy than the Sarma state at zero temperature.

From the definition, the superfluid density vanishes at the critical temperature $T_{c}$ for any imbalance $0<\alpha<$ $\alpha_{H S}$ and also at the other critical temperature $T_{o}>0$ when the imbalance is close to the maximum $\alpha_{H S}$. In weak coupling case, the chemical potentials $\mu_{\uparrow}$ and $\mu_{\downarrow}$ can be safely regarded as the Fermi energies of the two 
species, and near the critical temperatures $T_{c}$ and $T_{o}$ the superfluid density behaviors as 48]

$$
\begin{aligned}
& \rho_{s}=\rho_{c}\left(1-T / T_{c}\right)>0, \quad T \rightarrow T_{c}^{-}, \\
& \rho_{s}=\rho_{o}\left(1-T / T_{o}\right)<0, \quad T \rightarrow T_{o}^{+},
\end{aligned}
$$

where $\rho_{c}$ and $\rho_{o}$ depend only on the population imbal-
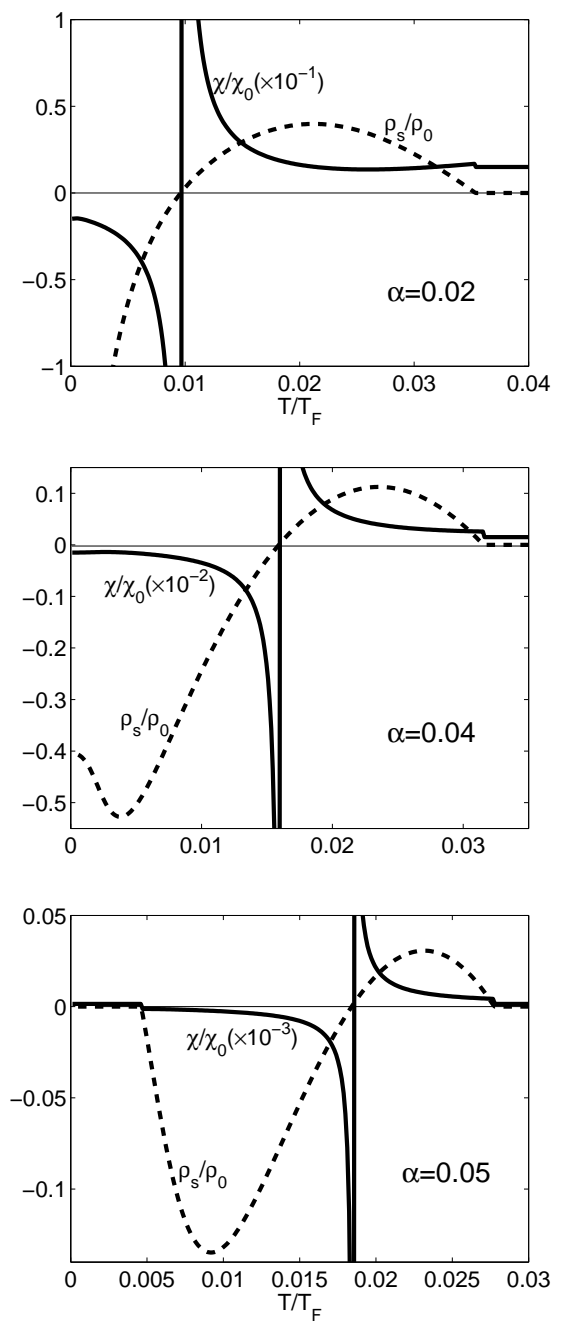

FIG. 2: The superfluid density $\rho_{s}$ (dashed lines), scaled by $\rho_{0}=m n$, and number susceptibility $\chi$ (solid lines), scaled by $\chi_{0}=n / E_{F}$, as functions of $T$, scaled by $T_{F}$, for three values of number asymmetry in the BCS regime with $p_{F} a_{s}=-0.6$.

ance $\alpha$. Up to this point, we have shown that the superfluid density is negative at low temperatures but should be positive near the critical temperature. In Fig 2 we show the superfluid density $\rho_{s}$ as a function of $T$ for different values of population imbalance $\alpha$. For the symmetric system with $\alpha=0, \rho_{s}$ is always positive in the whole superfluidity region. For systems with weak imbalance, $\rho_{s}$ is negative at low temperature, then changes sigh at an intermediate temperature $T_{1}$, and keeps to be positive at high temperature. When the imbalance is strong enough, $\rho_{s}$ is zero till the starting temperature $T_{o}$ of the superfluidity, then becomes negative first and turns to be positive at $T_{1}$. In Fig 3, we plot the two critical temperatures $T_{o}$ and $T_{c}$ and the intermediate temperature $T_{1}$ as functions of asymmetry parameter $\alpha$. The temperature $T_{1}$ separates the stable HS phase with $\rho_{s}>0$ at high temperature from the unstable HS phase with $\rho_{s}<0$ at low temperature. The negative superfluid density indicates that the LOFF state may be energetically flavored at low temperature, and there may be a phase transition from LOFF phase to HS phase at $T=T_{1}$. We will come back to this point in the next section.

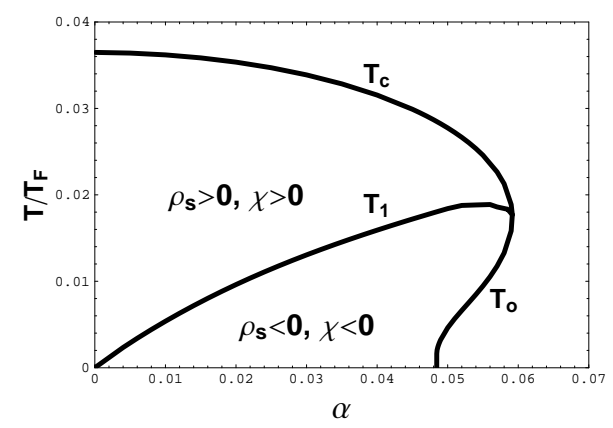

FIG. 3: The two critical temperatures $T_{o}$ and $T_{c}$ and the intermediate temperature $T_{1}$ as functions of the asymmetry parameter $\alpha$ in the BCS regime with $p_{F} a_{s}=-0.6 . \rho_{s}>$ $0, \chi>0$ and $\rho_{s}<0, \chi<0$ indicate, respectively, the stable and unstable HS phases.

\section{Number Susceptibility $\chi$}

The general stability condition for a two-component system against changes in the densities of its components is described by the total free energy of the system [50, 51], $F=\int d^{3} \mathbf{x} \mathcal{F}\left[n_{\sigma}(\mathbf{x})\right]$. Considering its fluctuations induced by small changes $\delta n_{\sigma}(\mathbf{x})$, the first-order variation $\delta F$ vanishes automatically due to the number conservation, $\int d^{3} \mathbf{x} \delta n_{\sigma}(\mathbf{x})=0$, and the second-order variation $\delta^{2} F$ is given by the quadratic form

$$
\delta^{2} F=\frac{1}{2} \int d^{3} \mathbf{x} \sum_{\sigma, \sigma^{\prime}=\uparrow, \downarrow} \frac{\partial^{2} \mathcal{F}}{\partial n_{\sigma} \partial n_{\sigma^{\prime}}} \delta n_{\sigma} \delta n_{\sigma^{\prime}}
$$

Therefore, to achieve a stable homogeneous phase, the $2 \times 2$ matrix $\partial^{2} \mathcal{F} / \partial n_{\sigma} \partial n_{\sigma^{\prime}}$ should be positively definite, namely, it has only positive eigen values. This matrix is hard to calculate since it is not easy to express explicitly the free energy as a function of densities. However, from the relation (13) between the free energy $\mathcal{F}\left(n_{\sigma}\right)$ and thermodynamic potential $\Omega\left(\mu_{\sigma}\right)$, it is easy to check that the stability condition to have positively definite matrix $\partial^{2} \mathcal{F} / \partial n_{\sigma} \partial n_{\sigma^{\prime}}$ is equivalent to the condition to have positively definite matrix $-\partial^{2} \Omega / \partial \mu_{\sigma} \partial \mu_{\sigma^{\prime}}$. 
For systems without mass difference between the two species, the condition to have positive eigenvalues of $-\partial^{2} \Omega / \partial \mu_{\sigma} \partial \mu_{\sigma^{\prime}}$ can be reduced to the constraint $[3]$ that the imbalance number susceptibility $\chi=-\left(\partial^{2} \Omega / \partial \delta \mu^{2}\right)_{\mu}=(\partial \delta n / \partial \delta \mu)_{\mu}$ should be positive. For $\chi<0$, the density difference $\delta n=n_{\downarrow}-n_{\uparrow}$ increases with decreasing chemical potential difference $\delta \mu$, which is certainly unphysical and means instability of the superfluid against the phase separation (PS). Employing the gap equation which determines the condensate as a function of chemical potentials, $\Delta=\Delta\left(\mu_{\sigma}\right)$, we can express the number susceptibility $\chi$ as a direct and indirect parts,

$$
\chi=\left(\frac{\partial \delta n}{\partial \delta \mu}\right)_{\mu, \Delta}+\left(\frac{\partial \delta n}{\partial \Delta}\right)_{\mu, \delta \mu}\left(\frac{\partial \Delta}{\partial \delta \mu}\right)_{\mu} .
$$

From the expression

$$
\delta n=\int \frac{d^{3} \mathbf{p}}{(2 \pi)^{3}}\left[f\left(E_{B}\right)-f\left(E_{A}\right)\right]
$$

which leads to

$$
\begin{aligned}
& \left(\frac{\partial \delta n}{\partial \delta \mu}\right)_{\Delta, \mu}=-\int \frac{d^{3} \mathbf{p}}{(2 \pi)^{3}}\left[f^{\prime}\left(E_{A}\right)+f^{\prime}\left(E_{B}\right)\right], \\
& \left(\frac{\partial \delta n}{\partial \Delta}\right)_{\mu, \delta \mu}=\int \frac{d^{3} \mathbf{p}}{(2 \pi)^{3}} \frac{\Delta}{E_{p}}\left[f^{\prime}\left(E_{B}\right)-f^{\prime}\left(E_{A}\right)\right],
\end{aligned}
$$

and the gap equation $(\partial \Omega / \partial \Delta)_{\mu, \delta \mu}=0$ which results in

$$
\left[\frac{\partial}{\partial \delta \mu}\left(\frac{\partial \Omega}{\partial \Delta}\right)_{\mu, \delta \mu}\right]_{\mu, \Delta}+\left(\frac{\partial^{2} \Omega}{\partial \Delta^{2}}\right)_{\mu, \delta \mu}\left(\frac{\partial \Delta}{\partial \delta \mu}\right)_{\mu}=0
$$

namely,

$$
\left(\frac{\partial \Delta}{\partial \delta \mu}\right)_{\mu}=\left(\frac{\partial \delta n}{\partial \Delta}\right)_{\mu, \delta \mu}\left(\frac{\partial^{2} \Omega}{\partial \Delta^{2}}\right)_{\mu, \delta \mu}^{-1},
$$

we have

$$
\chi=\left(\frac{\partial \delta n}{\partial \delta \mu}\right)_{\mu, \Delta}+\left(\frac{\partial \delta n}{\partial \Delta}\right)_{\mu, \delta \mu}^{2}\left(\frac{\partial^{2} \Omega}{\partial \Delta^{2}}\right)_{\mu, \delta \mu}^{-1} .
$$

Since $(\partial \delta n / \partial \delta \mu)_{\mu, \Delta}$ is always positive, the stability condition is controlled by the gap susceptibility $\kappa=$ $\left(\partial^{2} \Omega / \partial \Delta^{2}\right)_{\mu, \delta \mu}$ which determines if the solution of the gap equation is the minimum of the thermodynamic potential.

We now consider the relation between the gap susceptibility and the superfluid density. It is easy to explicitly write down the gap susceptibility,

$$
\begin{aligned}
\kappa= & \int \frac{d^{3} \mathbf{p}}{(2 \pi)^{3}} \frac{\Delta^{2}}{E_{p}^{2}}\left[\frac{1-f\left(E_{A}\right)-f\left(E_{B}\right)}{E_{p}}\right. \\
& \left.+\left(f^{\prime}\left(E_{A}\right)+f^{\prime}\left(E_{B}\right)\right)\right] \\
& +\frac{2}{g}-\int \frac{d^{3} \mathbf{p}}{(2 \pi)^{3}} \frac{1-f\left(E_{A}\right)-f\left(E_{B}\right)}{E_{p}},
\end{aligned}
$$

where the last line vanishes automatically due to the gap equation in the superfluid phase. In weak coupling limit, the number susceptibility and gap susceptibility in the superfluid phase at zero temperature can be evaluated as

$$
\chi=-\frac{3 n}{2 E_{F}}, \quad \kappa=\frac{m p_{F}}{\pi^{2}}\left[1-\frac{\delta \mu \theta(\delta \mu-\Delta)}{\sqrt{\delta \mu^{2}-\Delta^{2}}}\right]
$$

which are negative at any imbalance $\alpha$. On the other hand, applying partial integration to the number equations, the superfluid density can be expressed as

$$
\begin{aligned}
\rho_{s}= & \int \frac{d^{3} \mathbf{p}}{(2 \pi)^{3}} \frac{\mathbf{p}^{2}}{3} \frac{\Delta^{2}}{E_{p}^{2}}\left[\frac{1-f\left(E_{A}\right)-f\left(E_{B}\right)}{E_{p}}\right. \\
& \left.+\left(f^{\prime}\left(E_{A}\right)+f^{\prime}\left(E_{B}\right)\right)\right] .
\end{aligned}
$$

Comparing $\kappa$ and $\rho_{s}$ in the superfluid phase, the only difference is the factor of $\mathbf{p}^{2} / 3$ in the integrand function of $\rho_{s}$. In the BCS region, the integration is dominated by a narrow momentum window around the average Fermi momentum $p_{0}=\sqrt{2 m \mu}$, and we have

$$
\rho_{s} \simeq \frac{p_{0}^{2}}{3} \kappa \text {. }
$$

From this proportional relation between $\kappa$ and $\rho_{s}$ and note that $\kappa$ is the denominator of the second term of (29), while the superfluid density and number susceptibility have different amplitudes, they change sign almost at the same position. We have checked numerically that such an approximation holds well in the BCS region $-\infty<1 /\left(p_{F} a_{s}\right)<-1$. When $\rho_{s}$ changes from $\rho_{s}>0$ to $\rho_{s}<0$ at the turning temperature $T_{1}, \chi$ changes from positive infinity to negative infinity approximately at the same temperature. Therefore, when $\rho_{s}$ is not too large, namely when the second term of (29) is guaranteed to dominate $\chi, \rho_{s}$ and $\chi$ have almost the same positive and negative regions in the BCS limit. This temperature behavior of superfluid density and number susceptibility is clearly shown in Fig 2 where we presented $\rho_{s}$ and $\chi$ as functions of temperature. From the stability analysis at different population imbalance $\alpha$, we plot in Fig 3 the phase diagram of the homogeneous superfluid in the $T-\alpha$ plane for the coupling $p_{F} a_{s}=-0.6$. The temperature $T_{1}(\alpha)$ separates the stable HS phase at high temperature where $\chi$ and $\rho_{s}$ are positive from the unstable HS phase at low temperature where $\chi$ and $\rho_{s}$ are negative. Because of the divergence of $\chi$ at the turning point $T_{1}$, the possible phase transition from PS to HS must be of first order.

From the above analytic and numerical calculations in the BCS region, the conditions to guarantee a stable homogeneous superfluid described by the superfluid density, number susceptibility and gap susceptibility are equivalent. We can show that this is also true for unequal mass system. For instance, the breached pairing state proposed in 32] which is the minimum of the thermodynamic potential should also be stable against the LOFF 
state and PS state. Certainly, when the coupling is strong enough, the difference among these stable conditions will become remarkable. Due to the factor of $\mathbf{p}^{2} / 3$, the superfluid density is easier to be positive in strong coupling region than the number susceptibility, this is the reason why the authors in [3] found that the positive superfluid density is a weaker condition than the positive number susceptibility. At finite temperature, we found that for a strong enough coupling, the turning temperature $T_{1}$ for the superfluid density $\rho_{s}$ is remarkably different from the one for the number susceptibility $\chi$. Generally, the superfluid density $\rho_{s}$ becomes positive at a lower temperature, namely, there exists a large region where $\rho_{s}$ is positive but $\chi$ is negative. However, when the coupling is strong enough, the naive mean field treatment is not valid at finite temperature and we should employ some proper finite temperature theory for BCS-BEC crossover 44].

In the BCS region considered in this paper, from Figs 2 and 3, the LOFF and PS are both possible phases of the superfluid at low temperature $T<T_{1}$, the question left is to determine which of them is stable.

\section{LOFF PHASE VS HOMOGENEOUS PHASE}

We now take the LOFF state into account. Firstly we consider the LOFF state with $\alpha<\alpha_{H S}$ where the HS phase can survive. Before numerically solving the coupled gap equations (10) for $\Delta$ and $q$ together with the number equations (11) for $n_{\uparrow}$ and $n_{\downarrow}$, we discuss analytically the pair momentum in the temperature region close to the turning point $T_{1}$. The first order derivative of the thermodynamic potential with respect to the pair momentum can be written as

$$
\frac{\partial \Omega}{\partial q}=q W(q)
$$

which gives the gap equation for the pair momentum, $q W(q)=0$. The homogeneous and isotropic state corresponds to the trivial solution $q=0$, and the solution corresponding to the LOFF state is given by $W(q)=0$. From the second derivative,

$$
\frac{\partial^{2} \Omega}{\partial q^{2}}=W(q)+q W^{\prime}(q)
$$

and the relation between the superfluid density and the pair momentum susceptibility demonstrated in Section III, we have

$$
\rho_{s}=m^{2} W(0)
$$

which means

$$
W(0)=0
$$

at $T_{1}$ where $\rho_{s}$ changes sign continuously. Providing the LOFF solution is unique (we have checked this numerically at least in the BCS region), the LOFF momentum $q$ must vanish at $T_{1}$, and in the temperature region below and close to $T_{1}, q$ is very small and we have approximately the relation between the free energies for LOFF state and HS state,

$$
\mathcal{F}_{L O F F}=\mathcal{F}_{H S}+\frac{\rho_{s}}{2 m^{2}} q^{2}+O\left(q^{4}\right) .
$$

Therefore, in the unstable HS region with $\rho_{s}<0$ at $T \leq$ $T_{1}$, the LOFF state has lower free energy than the HS state, and the critical behavior of the LOFF momentum is

$$
q \sim\left(1-T / T_{1}\right)^{1 / 2}, \quad T \rightarrow T_{1}^{-}
$$

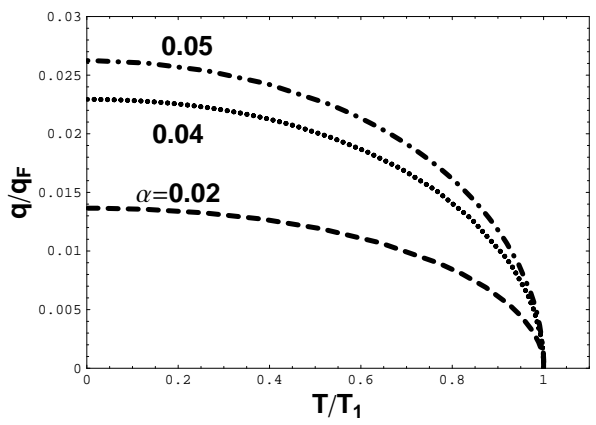

FIG. 4: The LOFF momentum $q$, scaled by the Fermi momentum $p_{F}$, as a function of $T$, scaled by the intermediate temperature $T_{1}$, for several values of number asymmetry $\alpha$.

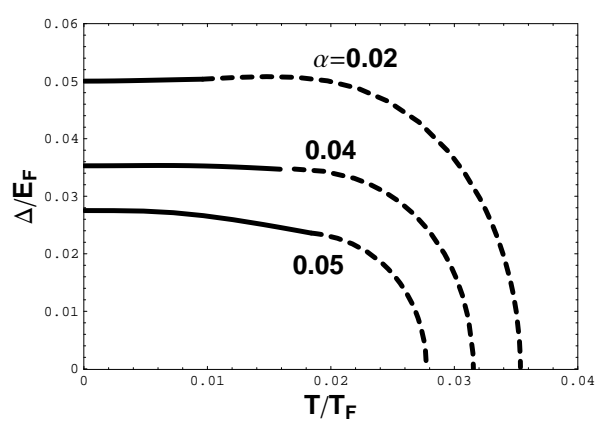

FIG. 5: The pairing gap $\Delta$, scaled by $E_{F}$, as a function of $T$, scaled by $T_{F}$, for several values of number asymmetry $\alpha$. The solid lines are for LOFF state and the dashed lines for BP state.

In Fig 4, we show the LOFF momentum $q$ scaled by the Fermi momentum $p_{F}$ as a function of temperature scaled by the intermediate temperature $T_{1}$ for several values of number asymmetry $\alpha$. At any $\alpha$, the momentum drops down from the maximum at $T=0$ to zero at $T=T_{1}$. Note that $T_{1}$ is $\alpha$ dependent, it increases with increasing asymmetry. In Fig 5 , we demonstrate the pairing gap $\Delta$ for the LOFF state (solid lines) and HS state (dashed lines). The LOFF state survives only in the region $0<$ 
$T<T_{1}$, and the stable HS state exists in the region $T_{1}<$ $T<T_{c}$. The two meet at the intermediate temperature $T_{1}$ with the continuity $\Delta_{L O F F}\left(T_{1}\right)=\Delta_{H S}\left(T_{1}\right)$. Different from the pair momentum which increases with increasing asymmetry, the gap parameter decreases with increasing asymmetry. It is necessary to point out that the LOFF state can survive not only in the unstable HS region, but also in the region where the HS phase disappears. For instance, for $\alpha=0.05$, the HS phase disappears in the region $0<T<T_{o}$, but $\mathrm{LOFF}$ state can survive there.
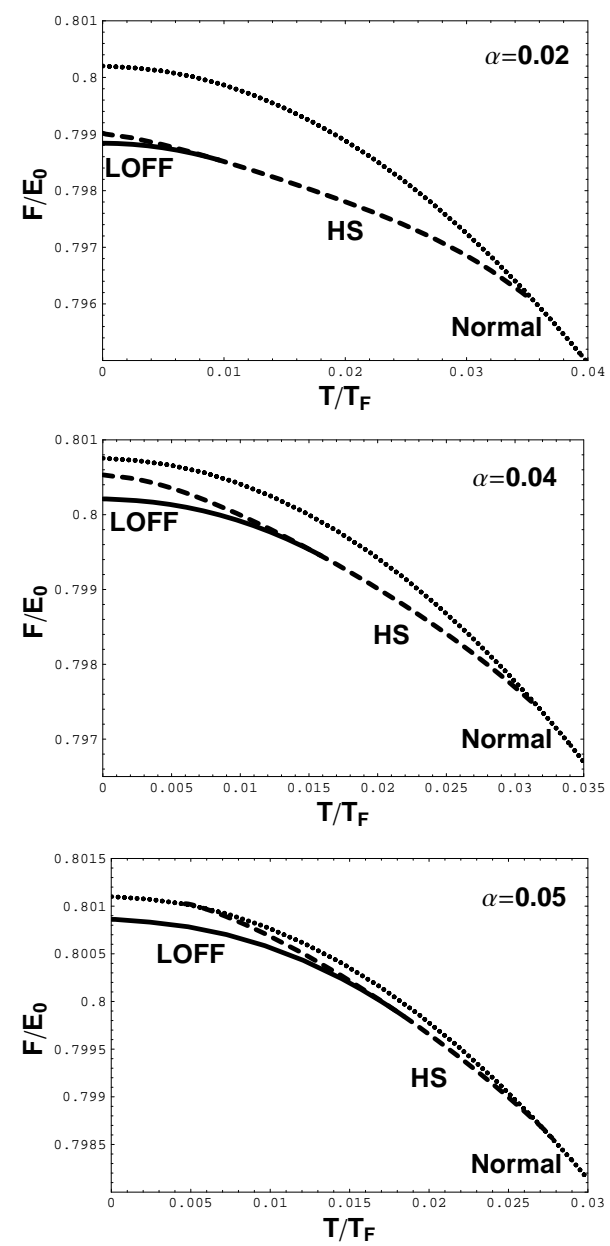

FIG. 6: The free energy $\mathcal{F}$, scaled by $E_{0}=p_{F}^{5} /\left(8 \pi^{2} m\right)$, as a function of $T$, scaled by $T_{F}$, for several values of number asymmetry $\alpha$. The solid, dashed and dotted lines correspond, respectively to the LOFF, HS and normal states.

In Fig 6 we compare the free energies for the LOFF, HS and normal state for different values of asymmetry $\alpha$. It is clear that LOFF is more stable than the other two in the region $0<T<T_{1}$, and HS is stable than the normal state in the region $T_{1}<T<T_{c}$. The phase transition from HS to LOFF happens at the intermediate temperature $T_{1}$, and the order parameter characterizing the spontaneous rotational symmetry breaking is the LOFF momentum $q$. Since $q$ drops down monotonously to zero when $T$ approaches to $T_{1}$, the phase transition is of second order. From the above result, the strange superfluidity window in the temperature region $T_{o}<T<T_{c}$ obtained in [44, 45] disappears, when the LOFF state is taken into account. The superfluid can exist in the whole temperature region $0<T<T_{c}$ with different phase at different temperature. When the temperature is higher than the critical value $T_{c}$, the strong thermal motion suppresses any superfluidity, and the system is in normal Fermi gas. From the continuous temperature behavior (16) of the HS gap, the phase transition at $T_{c}$ is of second order.

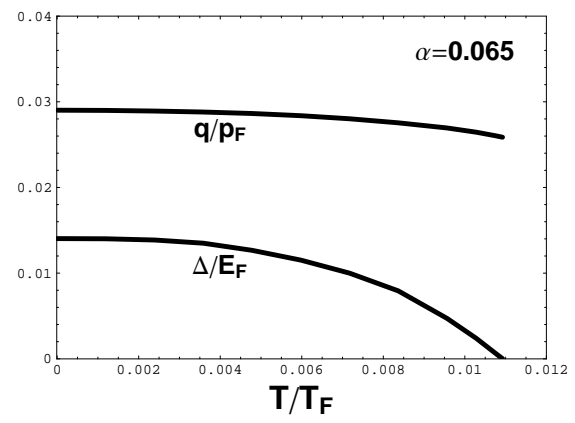

FIG. 7: The LOFF pairing gap $\Delta$, scaled by $E_{F}$, and the momentum, scaled by $p_{F}$, as functions of $T$, scaled by $T_{F}$, at $\alpha=0.065>\alpha_{H S}$.

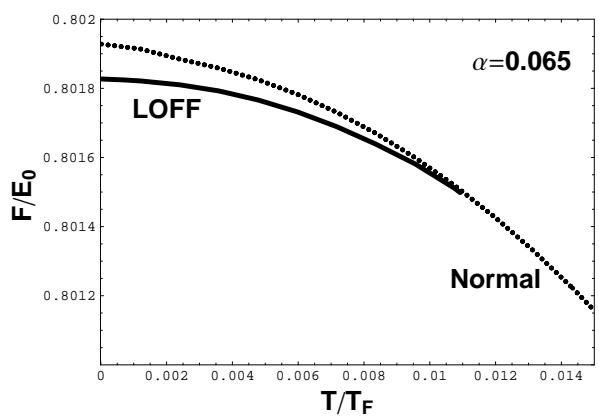

FIG. 8: The free energy $\mathcal{F}$, scaled by $E_{0}$, as a function of $T$, scaled by $T_{F}$, at $\alpha=0.065>\alpha_{H S}$. The solid and dotted lines are, respectively, for the LOFF and normal phases.

In the above discussion we have restricted ourself in the asymmetry region $\alpha<\alpha_{H S}$ where the HS phase can survive. What is about the case with $\alpha>\alpha_{H S}$, and can the LOFF further survive in high asymmetric systems where there is no room for the HS? In Fig:7 we show the LOFF momentum $q$ and gap $\Delta$ as functions of temperature for $\alpha=0.065>\alpha_{H S}$. Both drops down monotonously with increasing temperature, and the gap approaches to zero continuously. To determine the stable state, we compare the free energies for the LOFF and normal phase in Fig 8 . It is easy to see that the LOFF is stable at low temperature and the normal phase becomes the only possible state at high temperature, and 
the phase transition from the normal phase to LOFF is of second order. The fact that the LOFF can survive at higher density asymmetry where the HS disappears is consistent with the conclusion at zero temperature [43]. Certainly, when the asymmetry is beyond the maximum value $\alpha_{L O F F}$ for the LOFF state, the system is in the normal phase at any temperature.

\section{STABILITY OF LOFF PHASE}

In Section IV we neglected the possibility of the PS phase and focused only on the LOFF phase. Since both PS and LOFF phases can be the ground state in the temperature region $0<T<T_{1}$, we analyze in this section the stability of the known LOFF phase against the PS. To this end, we study again the imbalance number susceptibility $\chi=(\partial \delta n / \partial \delta \mu)_{\mu}$ for the LOFF state,

$$
\begin{aligned}
\chi= & \left(\frac{\partial \delta n}{\partial \delta \mu}\right)_{\mu, \Delta, q}+\left(\frac{\partial \delta n}{\partial \Delta}\right)_{\mu, \delta \mu, q}\left(\frac{\partial \Delta}{\partial \delta \mu}\right)_{\mu, q} \\
& +\left(\frac{\partial \delta n}{\partial q}\right)_{\mu, \delta \mu, \Delta}\left(\frac{\partial q}{\partial \delta \mu}\right)_{\mu, \Delta} .
\end{aligned}
$$

To simplify the expression, we ignore in the following the subscript notes and this will not make any confusion.

Similar to the calculation for the HS phase, from the number difference $\delta n$ (25), we obtain the same expression (26) for the derivatives $\partial \delta n / \partial \delta \mu$ and $\partial \delta n / \partial \Delta$ and the derivative

$$
\frac{\partial \delta n}{\partial q}=-\frac{1}{m} \int \frac{d^{3} \mathbf{p}}{(2 \pi)^{3}} \frac{\mathbf{p} \cdot \mathbf{q}}{q}\left[f^{\prime}\left(E_{A}\right)+f^{\prime}\left(E_{B}\right)\right] .
$$

With the derivatives $\partial \Delta / \partial \delta \mu$ and $\partial q / \partial \delta \mu$ evaluated from the coupled gap equations for $\Delta$ and $q$,

$$
\begin{gathered}
\frac{\partial^{2} \Omega}{\partial \Delta \partial \delta \mu}+\frac{\partial^{2} \Omega}{\partial \Delta^{2}} \frac{\partial \Delta}{\partial \delta \mu}+\frac{\partial^{2} \Omega}{\partial \Delta \partial q} \frac{\partial q}{\partial \delta \mu}=0 \\
\frac{\partial^{2} \Omega}{\partial q \partial \delta \mu}+\frac{\partial^{2} \Omega}{\partial q \partial \Delta} \frac{\partial \Delta}{\partial \delta \mu}+\frac{\partial^{2} \Omega}{\partial q^{2}} \frac{\partial q}{\partial \delta \mu}=0
\end{gathered}
$$

we have

$$
\chi=\frac{\partial \delta n}{\partial \delta \mu}+\frac{\left(\frac{\partial \delta n}{\partial \Delta}\right)^{2} \frac{\partial^{2} \Omega}{\partial q^{2}}+\left(\frac{\partial \delta n}{\partial q}\right)^{2} \frac{\partial^{2} \Omega}{\partial \Delta^{2}}-2 \frac{\partial \delta n}{\partial \Delta} \frac{\partial \delta n}{\partial q} \frac{\partial^{2} \Omega}{\partial q \partial \Delta}}{\operatorname{det} \mathcal{M}},
$$

where $\mathcal{M}$ is the stability matrix for the LOFF state defined as

$$
\mathcal{M}=\left(\begin{array}{cc}
\frac{\partial^{2} \Omega}{\partial \Delta^{2}} & \frac{\partial^{2} \Omega}{\partial \Delta \partial q} \\
\frac{\partial^{2} \Omega}{\partial q \partial \Delta} & \frac{\partial^{2} \Omega}{\partial q^{2}}
\end{array}\right)
$$

with the gap susceptibility $\partial^{2} \Omega / \partial \Delta^{2}$ given in (30) and the other two elements,

$$
\begin{aligned}
\frac{\partial^{2} \Omega}{\partial q^{2}}= & \frac{n}{m}+\frac{1}{m^{2}} \int \frac{d^{3} \mathbf{p}}{(2 \pi)^{3}}\left[\frac{(\mathbf{p} \cdot \mathbf{q})^{2}}{q^{2}}\left(f^{\prime}\left(E_{A}\right)+f^{\prime}\left(E_{B}\right)\right)\right. \\
& +2 \mathbf{p} \cdot \mathbf{q} \frac{\xi_{p}}{E_{p}}\left(f^{\prime}\left(E_{A}\right)-f^{\prime}\left(E_{B}\right)\right) \\
& +q^{2} \frac{\xi_{p}^{2}}{E_{p}^{2}}\left(f^{\prime}\left(E_{A}\right)+f^{\prime}\left(E_{B}\right)\right) \\
& \left.-q^{2} \frac{\Delta^{2}}{E_{p}^{3}}\left(1-f\left(E_{A}\right)-f\left(E_{B}\right)\right)\right] . \\
\frac{\partial^{2} \Omega}{\partial \Delta \partial q}= & \frac{1}{m} \int \frac{d^{3} \mathbf{p}}{(2 \pi)^{3}}\left[\frac{\mathbf{p} \cdot \mathbf{q}}{q} \frac{\Delta}{E_{p}}\left(f^{\prime}\left(E_{A}\right)-f^{\prime}\left(E_{B}\right)\right)\right. \\
& +q \frac{\Delta \xi_{p}}{E_{p}^{2}}\left(f^{\prime}\left(E_{A}\right)+f^{\prime}\left(E_{B}\right)\right) \\
& \left.+q \frac{\Delta \xi_{p}}{E_{p}^{3}}\left(1-f\left(E_{A}\right)-f\left(E_{B}\right)\right)\right] .
\end{aligned}
$$

The number susceptibility for the LOFF state is illustrated in Fig 9 as a function of temperature in the region $0<T / T_{1}<1$. For $\alpha<0.53 \alpha_{L O F F}, \chi$ is always negative in the whole region, which means unstable LOFF state. This instability of the LOFF state is consistent with the conclusion obtained in [10, 43] at zero temperature. For $0.53 \alpha_{L O F F}<\alpha<\alpha_{H S}, \chi$ is positive at low temperature, then changes sign at the turning temperature $T_{2}$, and keeps to be negative at higher temperature between $T_{2}$ and $T_{1}$. Similar to the number susceptibility in homogeneous case, it is divergent at the turning temperature $T_{2}$. When $\alpha$ is larger than the maximum asymmetry $\alpha_{H S}$ for the HS phase but less the maximum value $\alpha_{L O F F}$ for the LOFF phase, $\chi$ is always positive in the whole temperature region.

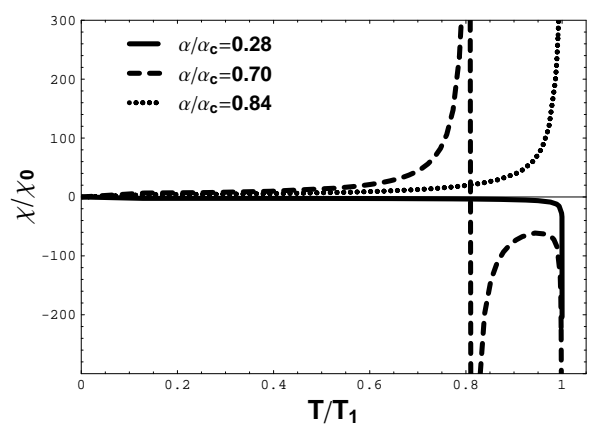

FIG. 9: The number susceptibility $\chi$ for the LOFF state, scaled by its value $\chi_{0}$ in the symmetric system with $\alpha=0$, as a function of temperature $T$, scaled by $T_{1}$, for several values of number asymmetry $\alpha$.

From the analytic and numerical calculations for the HS, LOFF, PS and normal state in this section and Sections [I, III and IV the ground states for the asymmetric system at different temperature and asymmetry are summarized in the phase diagram in $T-\alpha$ plane in Fig 10 . The dashed line separates the superfluid state at low 


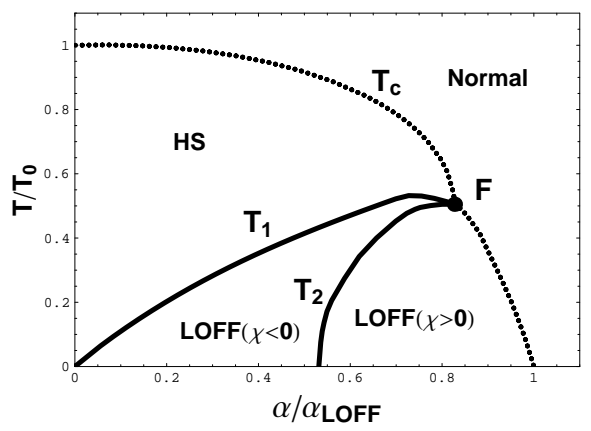

FIG. 10: The phase diagram in $T-\alpha$ plane. The solid lines $T_{1}$ and $T_{2}$ denote, respectively, the first order phase transitions from HS to PS and from LOFF to PS, the dashed line means the second order phase transition from HS or LOFF to normal phase, and F is the tetracritical point.

temperature and asymmetry from the normal state at high temperature and asymmetry. Inside the superfluid state, there are three phases, the homogeneous superfluid above the temperature $T_{1}$ marked by HS, the stable LOFF phase on the right hand side of the temperature line $T_{2}$ marked by LOFF $(\chi>0)$, and the unstable LOFF phase in between the temperature lines $T_{1}$ and $T_{2}$ marked by LOFF $(\chi<0)$ which is probably the PS phase. Since the number susceptibilities for HS and LOFF are both divergent at the corresponding turning temperatures $T_{1}$ and $T_{2}$, the phase transitions from LOFF to possible PS and from HS to PS are of first order. On the other hand, the phase transitions from HS to normal state and from LOFF to normal state are of second order. The four phases, HS, PS, LOFF and normal state meet at the point $\mathrm{F}$ located at $(T, \alpha)=\left(0.49 T_{0}, \alpha_{H S}=0.83 \alpha_{L O F F}\right)$ where $T_{0}$ is the critical temperature $T_{c}$ in the symmetric system with $\alpha=0$. The phase diagram we obtained here for the system with fixed densities is quite different from the one for the system with fixed chemical potentials 22, 33] in $T-\delta \mu$ plane. There is no room for the PS phase in the $T-\delta \mu$ plane, and the window for the LOFF and PS states in Fig 10 is much larger than the one for the LOFF state in $T-\delta \mu$ plane. While there exists a phase transition line from LOFF to HS in $T-\delta \mu$ plane, it disappears in the $T-\alpha$ plane.

We have checked in the frame of mean field approximation that the topological structure of the phase diagram in $T-\alpha$ plane does not change for a wide BCS region $-\infty<1 /\left(p_{F} a_{s}\right)<-1$. If pair fluctuation is included, the second order phase boundary between the normal phase and HS or LOFF phase will be shifted, but the topological structure will remain unchanged. Our phase diagram is similar to the one obtained in [17] based on the BdG formalism in an atomic trap.

While our result that the phenomenon of intermediate temperature superfluid shown in Fig [1 is washed out by introducing inhomogeneous pairing state is obtained by considering the simplest single plane wave LOFF state, the qualitative conclusion may remain unchanged when we take into account a more complicated LOFF state, since a general inhomogeneous state will produce a deeper minimum of the free energy of the system. On the other hand, we can restudy this problem by investigating the stability of the normal phase against an inhomogeneous fluctuation [20, 21, 52]. Let us consider a static but inhomogeneous pair fluctuation $\Phi(\mathbf{x})$ for the normal state in Fig 3 The effective action of the system can be expressed as a series of the fluctuation,

$$
S_{\text {eff }}=S_{0}+S_{2}+O\left(\Phi^{4}\right),
$$

where $S_{0}$ is the action of the normal Fermi gas. The quadratic term $S_{2}$ reads

$$
S_{2}=\frac{1}{2} \int \frac{d^{3} \mathbf{k}}{(2 \pi)^{3}} \mathcal{H}(\mathbf{k})|\tilde{\Phi}(\mathbf{k})|^{2},
$$

where $\tilde{\Phi}(\mathbf{k})$ is the Fourier transformation of $\Phi(\mathbf{x})$ and the function $\mathcal{H}$ is given by

$$
\mathcal{H}(\mathbf{k})=\frac{2}{g}-\sum_{\sigma} \int \frac{d^{3} \mathbf{p}}{(2 \pi)^{3}} \frac{1-f\left(\epsilon_{+}^{\sigma}\right)-f\left(\epsilon_{-}^{\sigma}\right)}{2 \epsilon_{\mu}+|\mathbf{k}|^{2} / 4 m}
$$

with $\epsilon_{\mu}=\epsilon_{p}-\mu$ and $\epsilon_{ \pm}^{\sigma}=(\mathbf{p} \pm \mathbf{k} / 2)^{2} /(2 m)-\mu_{\sigma}$. Note that $\mathcal{H}$ is an even function of $\mathbf{k}$, it depends only on the amplitude $k=|\mathbf{k}|, \mathcal{H}(k)$. For a symmetric system with $\alpha=0$, the sufficient and complete requirement for stable normal state is simply expressed as $\mathcal{H}(0)>0$ and the critical temperature is determined by $\mathcal{H}(0)=0$, because of the condition $\mathcal{H}(k) \geq \mathcal{H}(0)$. However, this requirement becomes incomplete for an asymmetric system with $\alpha \neq$ 0 , since $k=0$ is no longer the minimum of the function $\mathcal{H}(k)$. While the equality $\mathcal{H}(0)=0$ gives two critical temperatures $T_{o}$ and $T_{c}$, and the normal phase below $T_{o}$ is free from the ordinary BCS instability, characterized by $\mathcal{H}(0)>0$, we should demand $\mathcal{H}(k)>0$ for all $k$ to achieve a real stable normal state, and the critical temperature should be the maximum of those determined by $\mathcal{H}(k)=$ 0 . When we calculate the function $\mathcal{H}(k)$ in the normal phase, for the region close to the critical temperature $T_{o}$, $\mathcal{H}(k)$ is positive at $k=0$ but becomes negative in a range of nonzero $k$. This means that the normal phase there is stable against homogeneous superfluidity but unstable against inhomogeneous superfluidity. If we consider only homogeneous phases, we do find the strange intermediate temperature superfluidity. However, this is unrealistic when an inhomogeneous condensed phase enters.

\section{SUMMARY}

We have investigated the temperature behavior of the homogeneous and inhomogeneous superfluids in a twocomponent Fermi gas with density imbalance. The main conclusions are:

(1)For homogeneous superfluid, while the most favored pairing temperature is nonzero, and in the case with large 
asymmetry the superfluidity starts even at finite temperature, the superfluid density and the number susceptibility are negative at low temperature $T<T_{1}$. Therefore, the homogeneous superfluid is stable only at high temperature $T>T_{1}$ and unstable at low temperature $T<T_{1}$.

(2) The LOFF phase is energetically more favored than the homogeneous superfluid in the region $T<T_{1}$, and the phase transition from LOFF to homogeneous superfluid characterized by the pair momentum happens at $T_{1}$. Due to the existence of the inhomogeneous state at low temperature, the so called intermediate temperature superfluidity [23, 37, 44, 45] disappears and the pairing gap $\Delta$ is a monotonous function of the temperature.

(3)The phase separation is also energetically favored in the region where the homogeneous superfluid is unstable. From the calculation of the LOFF number susceptibility, the LOFF phase is stable only at large asymmetry, while the phase separation should be the ground state at small asymmetry.

(4)When the asymmetry is larger than the maximum value for the homogeneous superfluid and less than the limit for the LOFF superfluid, the homogeneous superfluid disappears at any temperature, but the LOFF superfluid survives and is stable at low temperature. When the asymmetry is too large, any superfluid vanishes, and the only possible state is the normal state.
(5)The phase transition from homogeneous superfluid or LOFF superfluid to normal state is of second order, and the transitions from phase separation to homogeneous superfluid and to LOFF superfluid are of first order. The four phases meet at the tetracritical point F. The obtained phase diagram in $T-\alpha$ plane with fixed densities is qualitatively different from the one in $T-\delta \mu$ plane with fixed chemical potentials.

We only analyzed the stability of homogeneous superfluid and LOFF superfluid against the phase separation and neglected the contribution from the surface energy. In further works a detailed calculation of the phase separation and the consideration of the surface energy [16, 53 . are needed.

Finally we should point out that our result obtained in the simple but general two-component model for atomic Fermi gas can be extended and applied to other physical systems, such as isospin asymmetric nuclear matter with neutron-proton pairing $23,24,25]$ and neutral color superconducting quark matter [27, 28].

Acknowledgement: The work was supported by the grants NSFC10428510, 10435080, 10575058 and SRFDP20040003103. We thank Q.Chen, H.Hu, M.Huang, T.Mizushima, H.Ren, M.Ruggieri and A.Sedrakian for valuable discussions.
[1] P.F.Bedaque, H.Caldas and G.Rupak, Phys. Rev. Lett. 91, 247002(2003); H.Caldas, Phys. Rev. A69, 063602(2004).

[2] A.Sedrakian, J.Mur-Petit, A.Polls and H.Mther, Phys.Rev. A72, 013613(2005).

[3] C.H.Pao, S.Wu and S.K.Yip, Phys. Rev. B73, 132506(2006).

[4] D.T.Son and M.A.Stephanov, Phys.Rev.A74, 013614(2006).

[5] D.E.Sheehy and L.Radzihovsky, Phys.Rev.Lett. 96, 060401(2006).

[6] K.Yang, cond-mat/0508484; cond-mat/0603190.

[7] J.Dukelsky, G.Ortiz and S.M.A. Rombouts, Phys.Rev.Lett 96, 180404 (2006).

[8] A.Bulgac, M.M.Forbes and A.Schwenk, Phys.Rev.Lett.97, 020402(2006).

[9] T.Ho and H.Zhai, cond-mat/0602568.

[10] X.Liu and H.Hu, Europhys.Lett.75, 364(2006); H.Hu and X.Liu, Phys.Rev.A73, 051603(R)(2006).

[11] Z.Gu, G.Warner and F.Zhou, cond-mat/0603091.

[12] M.Mannarelli, G.Nardulli and M.Ruggieri, Phys.Rev. A74, 033606(2006).

[13] T.Mizhushima, K.Machida and M.Ichioka, Phys.Rev.Lett.94, 060404(2005).

[14] J.Kinnunen, L.M.Jensen and P.Torma, Phys. Rev. Lett. 96, 110403(2006).

[15] P.Prieri and G.C.Strinati, Phys.Rev.Lett.96, 150404(2006)

[16] T.Silva and E.Mueller, Phys.Rev.A73, 051602(R)(2006); Phys.Rev.Lett. 97, 070402(2006).

[17] K.Machida, T.Mizushima and M.Ichioka,
Phys.Rev.Lett.97, 120407(2006).

[18] W. Yi, L.-M. Duan, Phys. Rev. A73, 031604(R)(2006).

[19] G.Sarma, J.Phys.Chem.Solid 24,1029(1963).

[20] A.I.Larkin and Yu.N.Ovchinnikov, Sov.Phys. JETP 20, 762(1965).

[21] P.Fulde and R.A.Ferrell,Phys. Rev 135, A550(1964).

[22] S.Takada and T.Izuyama, Prog.Theor.Phys.41,635(1969).

[23] A.Sedrakian and U.Lombardo, Phys. Rev. Lett. 84, 602(2000).

[24] A.I.Akhiezer, A.A.Isayev, S.V.Peletminsky, A.A.Yatsenko, Phys. Rev. C63, R021304(2000).

[25] A.Sedrakian, Phys.Rev. C63, 025801(2001).

[26] M.Huang, P.Zhuang, and W.Chao, Phys. Rev. D67, 065015(2003).

[27] I.Shovkovy and M.Huang, Phys. Lett.B564, 205(2003); Nucl.Phys.A729, 835-863(2003).

[28] M.Alford, C.Kouvaris and K. Rajagopal, Phys. Rev. Lett. 92, 222001(2004).

[29] H.Abuki, T.Kunihiro, Nucl.Phys. A768, 118(2006).

[30] D.Ebert and K.G.Klimenko, J.Phys.G32, 599(2006); L.He, M.Jin and P.Zhuang, Phys.Rev.D74, 036005(2006).

[31] W.V.Liu and F.Wilczek, Phys. Rev. Lett.90, 047002(2003).

[32] M.M.Forbes, E.Gubankova, W. Vincent Liu, F.Wilczek, Phys. Rev. Lett.94, 017001(2005).

[33] R.Casalbuoni and G.Nardulli, Rev.Mod.Phys. 76, 263(2004).

[34] M.W.Zwierlein, A.Schirotzek, C.H.Schunck and W.Ketterle, Science 311, 492(2006). 
[35] G.B.Partridge, W.Li, R.I.Kamar, Y.Liao and R.G.Hulet, Science, 311, 503(2006).

[36] Y.Shin, M.W.Zwierlein, C.H.Schunck, A.Schirotzek and W.Ketterle, Phys. Rev. Lett. 97, 030401 (2006).

[37] J.Liao and P.Zhuang, Phys. Rev. D68, 114016(2003).

[38] E.Gubankova, A.Schmitt and F.Wilczek, Phys.Rev.B74, 064505(2006).

[39] S.T.Wu and S.Yip, Phys.Rev. A67, 053603(2003).

[40] M.Huang and I.Shovkovy, Phys.Rev. D70, R051501(2004).

[41] L.He, M.Jin and P.Zhuang, Phys.Rev. B73, 024511(2006).

[42] I.Giannakis and H.Ren, Phys. Lett. B611, 137(2005).

[43] L.He, M.Jin and P.Zhuang, Phys.Rev.B73, 214527(2006).

[44] C.Chien, Q.Chen, Y.He and K. Levin, Phys.Rev.Lett.97,
090402(2006).

[45] A.Sedrakian, H.Muether and A.Polls, Phys.Rev.Lett.97, 140404(2006).

[46] J.-P. Martikainen, Phys.Rev.A74, 013602(2006).

[47] U. Lombardo, P. Nozires, P.Schuck, H.-J.Schulze and A. Sedrakian, Phys.Rev.C64, 064314(2001).

[48] A.L.Fetter and J.D.Walecka, Quantum Theory of ManyParticle Systems, McGraw-Hill, INC. 1971.

[49] W.V.Liu and F.Wilczek, cond-mat/0304632 S.Wu and S.Yip, cond-mat/0305675.

[50] L.Viverit, C.J.Pethick, and H.Smith, Phys. Rev. A61, 053605(2000).

[51] T.D.Cohen, Phys. Rev. Lett. 95, 120403(2005).

[52] H.Ren, hep-ph/0404074.

[53] H.Caldas, cond-mat/0601148. 